\title{
Correction to: Tobacco Use and Health-Related Quality of Life Among Individuals with Depression Who Are Receiving Treatment for HIV in Cape Town, South Africa
}

\author{
Amelia M. Stanton ${ }^{1,2,3}$. Jasper S. Lee ${ }^{4}$ Megan R. Wirtz ${ }^{3}$. Lena S. Andersen ${ }^{5} \cdot$ John Joska $^{5} \cdot$ Steven A. Safren ${ }^{4}$. \\ Richard van Zyl-Smit ${ }^{5}$. Conall O'Cleirigh ${ }^{1,2,3}$
}

Published online: 1 April 2021

(c) International Society of Behavioral Medicine 2021

\section{Correction to: https://doi.org/10.1007/ s12529-020-09951-z}

The original article has been updated to include the missing reference "Joska, J. A., Andersen, L. S., Smith-Alvarez, R., Magidson, J., Lee, J. S., O’Cleirigh, C., \& Safren, S. A. (2020). Nurse-delivered cognitive behavioral therapy for adherence and depression among people living with HIV (the Ziphamandla Study): protocol for a randomized controlled trial. JMIR research protocols, 9(2), e14200", and the corresponding citation has been included near the sentence "Participants in the present study completed the baseline assessment of a trial to treat clinical depression and ultimately improve ART adherence."
The original article can be found online at https://doi.org/10.1007/ s12529-020-09951-z.
Publisher's Note Springer Nature remains neutral with regard to jurisdictional claims in published maps and institutional affiliations.

\author{
Amelia M. Stanton \\ astanton2@mgh.harvard.edu \\ Jasper S. Lee \\ jslee@miami.edu \\ Lena S. Andersen \\ lena.andersen@uct.ac.za \\ John Joska \\ john.joska@uct.ac.za \\ Steven A. Safren \\ ssafren@miami.edu \\ Richard van Zyl-Smit \\ richard.vanzyl-smit@uct.ac.za
}

Department of Psychiatry, Massachusetts General Hospital, One Bowdoin Square, Suite 761, Boston, MA 02114, USA

2 The Fenway Institute, Fenway Health, 1340 Boylston Street, Boston, MA 02115, USA

3 Department of Psychiatry, Harvard Medical School, Boston, MA 02114, USA

4 Department of Psychology, The University of Miami, P.O. Box 248185, Coral Gables, FL 33124, USA

5 Department of Psychiatry, The University of Cape Town, Groote Schuur Drive, Observatory, Cape Town 7925, South Africa 